\section{Haemotoxic snakebite in rural \\ KwaZulu-Natal, South Africa}

To the Editor: We would like to comment on the case report published in the May 2016 issue of $S A M J^{[1]}$ of a suspected boomslang bite presenting with severe haematemesis. We question the diagnosis of a boomslang bite.

Firstly, the clinical manifestations as described do not correlate with a boomslang bite. Boomslang bite results in severe consumptive coagulopathy with bleeding from multiple sites and would not be confined to a single organ system as described in the case report. Haemorrhagic gastritis and ulcers also do not fit the clinical picture. These findings may be better explained by chronic heavy alcohol consumption.

Secondly, the normal international normalised ratio (INR) does not correlate with a coagulopathy. Furthermore, it should be emphasised that the polyvalent antivenom is not effective in the management of boomslang bite. We recommend the easy-to-perform bedside whole-blood clotting time investigation to diagnose boomslang bite in resource-limited areas. ${ }^{[2]}$ Place $5 \mathrm{~mL}$ of whole blood in a clean (preferably new) dry test tube at room temperature. At 20 minutes, tip the tube upside down once. If no clot is seen, the test is positive, indicating abnormal coagulation. If any clot (even very small) is observed, the test is negative. Alternatively, the syringe method can also be used. Draw $5 \mathrm{~mL}$ of whole blood in a plastic syringe. Leave the syringe untouched on a flat surface and tilt after 20 minutes to observe whether or not the blood has clotted. The bedside wholeblood clotting time could be repeated 4-hourly after a suspected bite, as the coagulopathy may develop late (12 - 36 hours after boomslang bite).

\section{E H Decloedt, G J Müller}

Division of Clinical Pharmacology, Department of Medicine, Faculty of Medicine and Health Sciences, Stellenbosch University, Cape Town, South Africa

ericdecloedt@sun.ac.za

1. Wagener M. Haemotoxic snakebite in rural KwaZulu-Natal, South Africa: A case presenting with haematemesis. S Afr Med J 2016;106(5):459-460. DOI:10.7196/SAMJ.2016.v106i5.9124

2. Punguyire D, Iserson KV, Stolz U, Apanga S. Bedside whole-blood clotting times: Validity after snakebites. J Emerg Med 2013;44(3):663-667. DOI:10.1016/j.jemermed.2012.07.073

S Afr Med J 2016;106(8):743. DOI:10.7196/SAMJ.2016.v106i8.11119 